\title{
Management of hyponatremia
}

\author{
Jennifer Ji Young Lee BHSc, Kajiru Kilonzo MD, Amy Nistico MD, Karen Yeates MD
}

$\mathrm{H}$ yponatremia, defined as a decrease in serum sodium below $136 \mathrm{mmol} / \mathrm{L}$, is a common occurrence in both inpatients and outpatients and can be found in up to $15 \%$ of the general hospital populations. ${ }^{1,2}$ Acute hyponatremia (duration $<48 \mathrm{~h}$ ) and its management can be a cause of major morbidity and mortality among patients in hospital. ${ }^{3}$ Chronic hyponatremia (duration $>48 \mathrm{~h}$ ), which is typically seen among older ( $\geq 65 \mathrm{yr}$ ) outpatients, also contributes to morbidity because it is often unrecognized. ${ }^{3,4}$ Typically, correction of serum sodium concentration in patients with acute hyponatremia should occur at a more rapid rate than in patients with chronic hyponatremia because the symptoms are usually more severe when serum sodium rapidly decreases over a short period. ${ }^{1}$

It is essential to determine the underlying cause of hyponatremia because the type of hyponatremia dictates the approach to therapy. Once the type is determined, clinicians need to weigh the evidence to determine what treatment will be the most effective. This can prove confusing and challenging for both primary care physicians and specialists because there are a myriad of treatment-related reviews and publications with no clear guide as to what the best evidence suggests for the treatment of hyponatremia in each setting.

In this review, we discuss the evidence for the management of hyponatremia. A variety of management approaches (both pharmacologic and nonpharmacologic) exist in clinical practice. We set out to review the evidence from randomized controlled trials (RCTs) for strategies to treat hyponatremia. Not surprisingly, most RCT-level evidence comes from industry-sponsored trials of newer pharmacologic agents. ${ }^{5,6}$

Our review was performed systematically; our methods are described in Box 1. After completing the review, it became clear that to provide the reader with a clinical approach that could be applied in practice, we also needed to review and discuss management strategies that are well established in practice but are not supported by RCT-level evidence. The clinical application of our results is shown in Box 2.

\section{How does the type of hyponatremia affect treatment?}

Although there are many approaches to the management of hyponatremia, one of the most common approaches used by nonexpert clinicians begins with an assessment of extracellular fluid (ECF) volume status. Because diabetes mellitus is becoming more prevalent in the adult population, clinicians should be mindful of ruling out hyperosmolar hyponatremia caused by hyperglycemia as a potential cause of hyponatremia. The clinical history and physical examination provide important diagnostic clues that help to classify the cause of hyponatremia as hypovolemic, euvolemic or hypervolemic. ${ }^{8}$

\section{Hypovolemic hyponatremia}

Hypovolemic hyponatremia occurs in cases of volume contraction, such as is seen with vomiting and diarrhea, excessive sweating and use of diuretics. ${ }^{1}$ A patient may present with frank hypotension or with postural hypotension or tachycardia. In this setting, release of both antidiuretic hormone (ADH) (increases renal permeability to water) and renin (increases renal sodium retention) occurs. Suspected hypovolemic hyponatremia may be confirmed by a urine sodium concentration below $20 \mathrm{mmol} / \mathrm{L}$, urine osmolality greater than $500 \mathrm{mOsm} / \mathrm{kg} \mathrm{H}_{2} \mathrm{O}$ and, typically, low urine volume. However, clinicians should be aware that patients with diuretic use may not have a low urine sodium level
Competing interests:

Karen Yeates has received honoraria for a career mentorship talk from Amgen and for a continuing medical education event from Otsuka

Pharmaceuticals. No other competing interests were declared.

This article has been peer reviewed.

Correspondence to:

Karen Yeates,

yeatesk@queensu.ca

CMAJ 2014. DOI:10.1503 /cmaj.120887

\section{- Ker poInTS}

- Hyponatremia is common and can be challenging to manage.

- Evaluation of the patient's fluid status is important in determining the type of hyponatremia, which will direct the type of management required.

- Limited evidence exists for many of the interventions commonly used to treat hyponatremia.

- Evidence from randomized controlled trials is largely centred around studies of vasopressin receptor antagonists with surrogate outcome measures.

- Rapid correction of hyponatremia is particularly dangerous in the setting of chronic hyponatremia. It should be carried out in a monitored setting with close observation of serum sodium levels. 
despite being hypovolemic. In addition, these patients may exacerbate their hyponatremia through the ingestion of solute-poor fluids (e.g., water or tea). ${ }^{9}$ The most common treatment option proposed for patients with hypovolemic hyponatremia is replacement of both salt and water through the intravenous infusion of sodium chloride solutions. ${ }^{9-11}$ Our review did not reveal any head-to-head comparison trials of different methods or types of intravenous fluids for the treatment of hypovolemic hyponatremia. In practice, infusion with normal saline (9\% sodium) is recommended to restore ECF volume by replacing both salt and free water., ${ }^{1,2}$

\section{Box 1: Identification of studies}

We performed a systematic review on the management of hyponatremia through searches of Ovid Medline (1946 through June 2011), Embase (1947 through July 2011) and the Cochrane database. Our initial search identified terms relevant to hyponatremia and included "hyponatremia" or "inappropriate ADH syndrome" or "water intoxication" or "Schwartz Barter syndrome." To identify relevant study designs, we searched the terms "randomized controlled trials" (RCTs) and initially included the search terms "cohort study" and "case-control study" out of interest to see how many nonRCT studies existed in the literature. We conducted our search with the assistance of a librarian who specializes in literature searches. We included studies written in English, that included patients aged 18 years and older and for whom the diagnosis of hyponatremia was established based on volume status and laboratory assessment with a serum sodium level less than $135 \mathrm{mmol} / \mathrm{L}$. We excluded expert opinions, literature reviews and studies that involved medications not used in North America. Two independent reviewers (K.Y. and J.J.L.) screened the titles and abstracts of the studies for eligibility based on our inclusion and exclusion criteria. The primary outcome was change in serum sodium level. The secondary outcomes were deaths or adverse reactions to the intervention. We followed the Preferred Reporting Items for Systematic reviews and Meta-Analyses (PRISMA) statement as closely as possible. ${ }^{7}$ Two additional trials published after July 2011 were added to our review after the original search took place. ${ }^{5,26}$ Detailed methods are described in Appendix 1 (available at www.cmaj.ca/lookup/suppl/doi:10.1503/cmaj.120887//DC1). The quality of the identified studies was mixed (Appendix 2, available at www.cmaj.ca/lookup/suppl/doi:10.1503/cmaj.120887/-/DC1).

\section{Box 2: Applying the results of this review in clinical practice}

A 60-year-old man presents with increasing dyspnea on exertion. He has known coronary artery disease and a history of congestive heart failure, currently managed with $120 \mathrm{mg} / \mathrm{d}$ of furosemide taken orally. His blood pressure is 100/60 $\mathrm{mm} \mathrm{Hg}$, his jugular venous pressure is $6 \mathrm{~cm}$ above the sternal angle, he has marked pitting edema of the lower limbs, and he has crackles at his lung bases. His serum sodium level is $125 \mathrm{mmol} / \mathrm{L}$.

This patient has hypervolemic hyponatremia as a result of decompensated congestive heart failure. His urine sodium concentration is $114 \mathrm{mmol} / \mathrm{L}$. The standard of care includes both water restriction and salt restriction, despite a lack of high-quality evidence to support this intervention. He will require loop diuretic therapy (e.g., furosemide) to treat extracellular fluid volume overload.

The randomized trial evidence evaluated in our review suggests that his serum sodium level could be corrected through the induction of aquaresis with the use of a vasopressin receptor antagonist. However, the lack of patient-focused outcomes makes it less clear as to what the true benefit to this patient would be in the long-term. If the choice to use a vasopressin receptor antagonist is made, we recommend that this be carried out in a monitored acute care setting under the guidance of a physician who has experience with its use.

\section{Euvolemic hyponatremia}

Euvolemic hyponatremia is divided into disorders associated with inappropriate stimulation of $\mathrm{ADH}$ release and those that are not. To determine whether a patient who has euvolemic hyponatremia has a disorder of excess water or a disorder due to inappropriate levels of $\mathrm{ADH}$, clinicians should measure urine sodium and urine osmolality. If the patient has syndrome of inappropriate $\mathrm{ADH}$ release (SIADH), the urine is usually inappropriately concentrated $\left(>100 \mathrm{mOsm} / \mathrm{kg} \mathrm{H}_{2} \mathrm{O}\right.$ ). Urine sodium concentration is usually greater than $30 \mathrm{mmol} / \mathrm{L}$. In disorders of excess water intake, the urine osmolality is usually less than $100 \mathrm{mOsm} / \mathrm{kg} \mathrm{H}_{2}$. Beer-drinker potomania (the ingestion of solute-poor fluid), psychogenic polydipsia (the consumption of large volumes of water in a short period), adrenal insufficiency and hypothyroidism are all causes of euvolemic hyponatremia that are not associated with inappropriate release of $\mathrm{ADH}$. Treatment of these underlying conditions or cessation of the contributing behaviour results in resolution of hyponatremia ${ }^{10,12,13}$

\section{Hypervolemic hyponatremia}

Hypervolemic hyponatremia is usually a result of increased ECF volume such as that seen with congestive heart failure, cirrhosis and nephrotic syndrome. Patients with these conditions tend to have low effective circulating volume that is sensed by the kidney and leads to avid sodium and water retention. The result is disproportionately elevated total body water in comparison to body sodium, and symptomatic volume overload (e.g., edema, ascites and dyspnea). The urine sodium level is typically lower than $20 \mathrm{mmol} / \mathrm{L}$, unless the patient is taking a diuretic (which leads to natriuresis). ${ }^{1,914}$ The care of such patients has historically included water restriction to $1-1.5 \mathrm{~L} / \mathrm{d}$ and a low-salt diet. ${ }^{9,10}$ Loop diuretics (e.g., furosemide) have been suggested to promote renal salt and water excretion. ${ }^{14}$ Involvement of cardiology, hepatology and nephrology specialists can be sought for optimal management in the respective settings. Vasopressin receptor antagonists have been used in clinical trials involving hypervolemic patients with hyponatremia.

\section{What are the current recommended therapies for hyponatremia in patients with syndrome of inappropriate ADH release?}

Syndrome of inappropriate ADH release is a clinical diagnosis suspected in patients with euvolemic hyponatremia who do not have one of the diagnoses mentioned above (e.g., beer-drinker poto- 
mania, psychogenic polydipsia, adrenal insufficiency). There are many causes of SIADH, including malignant disease, intracranial pathology and some medications. SIADH is a diagnosis of exclusion and implies normal renal, thyroid and adrenal function..$^{15}$ Proposed treatment of SIADH includes the management of the underlying disorder or discontinuation of the offending medication. However, reversal of the initiating disorder is not always possible. In such cases, and while the underlying cause is being evaluated, current therapy is to restrict the patient's intake of fluid to less than 1-1.5 L/d. ${ }^{11}$ Despite fluid restriction, some patients with SIADH require additional therapeutic regimens; these have varying degrees of efficacy and are not all supported by RCT-level evidence. These include demeclocycline, loop diuretics in combination with increased salt intake and vasopressin receptor antagonists. ${ }^{1,1,1,14,16}$

Demeclocycline, a tetracycline that inhibits the intracellular effects of $\mathrm{ADH}$ on renal tubular cells, can be used at a dose of $600 \mathrm{mg} / \mathrm{d} .{ }^{16}$ Alexander and colleagues ${ }^{17}$ conducted a double-blind, placebo-controlled crossover trial involving nine patients with a chronic psychiatric illness and documented hyponatremia. Demeclocycline was administered for 21 days, and serum sodium levels were obtained. Compared with placebo, sodium levels in the treatment group were not statistically different. We identified no other eligible studies in our search.

Some authors suggest that high salt intake combined with furosemide may be used to impair renal tubular responsiveness to ADH. It is suggested that this can be accomplished with a dose of $20 \mathrm{mg} / \mathrm{d}$ of furosemide. ${ }^{18}$ Clinicians should be mindful that the administration of a $0.9 \%$ sodium chloride solution intravenously usually worsens the degree of hyponatremia in patients with SIADH. This is caused by the hypo-osmolarity of normal saline (osmolality of $308 \mathrm{mOsm} / \mathrm{kg} \mathrm{H}_{2} \mathrm{O}$ ) compared with the urine osmolality in patients with SIADH (usually $>450 \mathrm{mOsm} / \mathrm{kg} \mathrm{H}_{2} 0$ ). As a result, the net fluid shift is into the intravascular space, which worsens hyponatremia.

\section{What is the role of vasopressin receptor antagonists in treating hyponatremia?}

Vasopressin receptor antagonists (often called the "vaptan" class of drugs) inhibit ADH by binding to renal vasopressin receptors and inducing aquaresis, thereby allowing a controlled increase in the serum concentration of sodium through the excretion of free water instead of both salt and water combined. Our search found six clinical trials of conivaptan, six trials of tolvaptan, three trials of satavaptan and one trial of lixivaptan. The characteristics of each trial are shown in Appendix 3 (available at www.cmaj.ca /lookup/suppl/doi:10.1503/cmaj.120887/-/DC1).

Vasopressin receptor antagonists have been studied in the setting of both hypervolemic and euvolemic hyponatremia (e.g., SIADH). There are several RCTs comparing vaptans to placebo for the treatment of SIADH. Most trials also involved patients with hypervolemia and therefore makes their applicability to patients with euvolemic SIADH more difficult. One study of the use of vaptans that involved 34 patients diagnosed with SIADH reported some improvement in the serum concentrations of sodium with the administration of satavaptan. ${ }^{19}$ At present, only tolvaptan is licensed in Canada under the indication of "clinically important nonhypovolemic hyponatremia."

In our review, we found one $\mathrm{RCT}^{20}$ that compared tolvaptan (a vaptan) to fluid restriction. The primary outcome was normalization of serum sodium or a $10 \%$ increase from baseline. At the end of the trial, tolvaptan appeared to be slightly more effective than fluid restriction in the treatment of hyponatremia. The limitations of this study were its small size, the mixture of patients with both euvolemic and hypervolemic hyponatremia and the use of serum sodium as the outcome as compared with using a more clinically relevant outcome based on signs or symptoms. Based on these limitations, the utility of tolvaptan or other vaptans in the setting of euvolemic hyponatremia is still unclear. No RCTs of fluid restriction compared with other interventions were found.

\section{Conivaptan}

Conivaptan is a novel antagonist of AVP V1A/V2 receptors. ${ }^{20}$ We identified six RCTs. ${ }^{21-26}$ Annane and colleagues ${ }^{23}$ and Ghali and colleagues $^{27}$ investigated the efficacy of oral conivaptan; the remainder focused on the use of intravenous administration in patients with euvolemic or hypervolemic hyponatremia. Both oral conivaptan studies (using doses ranging from $40 \mathrm{mg} / \mathrm{d}$ to $80 \mathrm{mg} / \mathrm{d}$ ) showed significant efficacy in correcting sodium levels compared with those in the placebo group. Ghali and colleague ${ }^{27}$ also reported that the median time to achieve an increase in serum sodium of $4 \mathrm{mmol} / \mathrm{L}$ or more above baseline was significantly shorter in the conivaptan groups than in the placebo group.

Conivaptan is not available for use in Canada. The United States Food and Drug Administration allows the use of a loading dose $(20 \mathrm{mg})$ given intravenously over 30 minutes followed by a continuous infusion of $20-40 \mathrm{mg} / \mathrm{d}$ for patients admitted to hospital with euvolemic or hyper- 
volemic hyponatremia. ${ }^{21,22}$ Verbalis and colleauges ${ }^{22}$ evaluated the efficacy and tolerability of conivaptan infusions and found that both $40 \mathrm{mg} / \mathrm{d}$ and $80 \mathrm{mg} / \mathrm{d}$ raised serum sodium levels over the course of four days and were generally well tolerated by 56 patients. Similar findings were observed for 84 patients in the study by Zeltser and colleagues, ${ }^{25}$ which also found that the differences between the doses were not statistically significant.

Conivaptan was considered safe and well tolerated in most studies. The most commonly reported adverse effects with intravenous administration were infusion-site reactions, such as erythema and pain. ${ }^{21,25}$ More infusion-site reactions were seen with higher conivaptan doses..$^{24,25}$

\section{Tolvaptan}

Tolvaptan is the only vaptan available in Canada and is a selective oral antagonist of the V2 receptor, causing a dose-dependent increase of dilute urine. ${ }^{26,28}$ We identified six RCTs in our review. ${ }^{5,6,20,29-31}$ Tolvaptan has been used for euvolemic and hypervolemic hyponatremia. Two randomized, placebo-controlled, double-blind phase three clinical trials (SALT-1 and SALT-2; $n=448$ ) showed the effectiveness of oral tolvaptan compared with placebo in increasing serum sodium concentrations within 30 days of therapy in patients with congestive heart failure, cirrhosis or SIADH. ${ }^{31}$ The trials found that those in the tolvaptan group (doses ranged from $15 \mathrm{mg} / \mathrm{d}$ to $60 \mathrm{mg} / \mathrm{d}$ ) had significantly higher serum sodium concentrations than those in the placebo group from the time of the first treatment to the last treatment, and the normal serum sodium range was reached more rapidly. After stopping the drug, there was no statistical difference in the decline of serum sodium between the two groups. Verbalis and colleagues ${ }^{30}$ and Cárdenas and colleagues ${ }^{5}$ analyzed the SIADH and cirrhosis subgroups, respectively, within the SALT trials and found similar results. A four-year openlabel extension study of the SALT trials, known as SALTWATER, found that the increases in serum sodium levels were maintained over longer periods of time. ${ }^{31}$ Josiassen and colleagues ${ }^{29}$ focused specifically on idiopathic hyponatremia in patients with schizophrenia and reported similar findings. Gheorghiade and colleagues ${ }^{20}$ showed in a small trial $(n=28)$ that tolvaptan was superior to fluid restriction in the correction of serum sodium levels after 27 days of treatment and over a follow-up of 65 days.

Tolvaptan was also considered safe and well tolerated in studies. Adverse events include thirst or dry mouth, ${ }^{28,29}$ ECF volume contraction, ${ }^{26,29}$ hypotension, ${ }^{26,29}$ urinary tract infections, hyper- glycemia, hyperkalemia, dizziness and headaches. ${ }^{29}$ Only $4 \%$ of participants in the SALT trials withdrew because of adverse events, and the number of deaths was similar in the placebo and treatment groups. ${ }^{31}$

\section{Satavaptan}

Satavaptan is an oral, highly selective, V2 receptor antagonist. It has been studied in patients with SIADH, ${ }^{19}$ cirrhosis with ascites ${ }^{32}$ and, most recently, congestive heart failure.$^{33}$ All studies have reported a beneficial effect on the level of serum sodium. Soupart and colleagues ${ }^{19}$ assessed the short-and long-term effectiveness of the drug in patients with SIADH of various origins and found that the significant increase in sodium levels compared with placebo were maintained over 12 months of treatment. Ginès and colleague's study $^{32}$ involved 110 patients with cirrhosis and ascites; they reported that satavaptan significantly improved ascites control, reduced abdominal girth and improved sodium levels. Aronson and colleagues ${ }^{33}$ found that a dose of $50 \mathrm{mg}$ had a significantly higher response rate compared with placebo. A 25-mg dose was not found to have a clinically significant effect, although for patients with congestive heart failure, there appeared to be some effect.

Adverse events reported included thirst, atrial fibrillation, prolonged QTc intervals, hypotension, hypertension and pyrexia. More adverse events were reported with higher doses. ${ }^{33}$

\section{Lixivaptan}

One study assessed the efficacy and safety of lixivaptan, a selective oral vasopressin V2 receptor antagonist, in patients with cirrhosis and hypervolemic hyponatremia. Gerbes and colleagues ${ }^{34}$ found that lixivaptan was associated with a dosedependent response $(100 \mathrm{mg} / \mathrm{d}$ v. $200 \mathrm{mg} / \mathrm{d})$ in normalization of serum sodium levels. Reported adverse outcomes included thirst, dehydration and renal impairment (increase of serum creatinine $>200 \mathrm{umol} / \mathrm{L}$ ).

A large clinical trial known as THE BALANCE study was recently conducted involving 650 patients admitted to hospital with heart failure and hyponatremia. Only the clinical trial design has been presented, ${ }^{35}$ and the publication of results are awaited.

\section{When should clinicians administer hypertonic saline?}

Any patient who presents with neurologic compromise or seizures as a result of hyponatremia requires urgent correction of serum sodium level, 
regardless of the rate of the fall in sodium. Correction is accomplished by use of intravenous hypertonic sodium chloride, usually at a concentration of $3 \%$, at $1-2 \mathrm{~mL} / \mathrm{kg} / \mathrm{h}$ in a monitored setting. The cause of hyponatremia should be determined from the patient's history and physical exam and should be managed based on volume status.,

We found one preliminary RCT comparing the oral and intravenous administration of hypertonic saline $(3 \%)$ in ultramarathon runners with asymptomatic exercise-associated hyponatremia. ${ }^{36}$ This study found that prompt intravenous administration of a $100 \mathrm{~mL}$ bolus of $3 \%$ saline was associated with normalization of serum sodium concentration, whereas no similar effect was seen for the same dose given orally.

\section{What are the roles of other less common therapies such as hydrocortisone, demeclocycline and desmopressin acetate in hyponatremia?}

We identified one RCT that compared hydrocortisone to placebo. Hyponatremia due to excessive natriuresis is common in patients with subarachnoid hemorrhages. Hydrocortisone has been shown to promote sodium retention in the kidney. Katayama and colleagues ${ }^{37}$ randomly assigned 71 patients with subarachnoid hemorrhage to placebo or $1200 \mathrm{mg} / \mathrm{d}$ of hydrocortisone for 10 days. The primary outcome in this study was the prevention of hyponatremia. Hydrocortisone was shown to maintain sodium serum levels throughout 14 days and prevented excess sodium secretion. However, hydrocortisone did not significantly reduce the incidence of symptomatic cerebral vasospasm nor did it improve overall outcomes. Given the lack of improvement in overall outcomes and the specific nature of the use of hydrocortisone in this study (patients with subarachnoid hemorrhage), we cannot safely recommend that it be used in the general population of patients who present with hyponatremia.

As previously discussed, our search yielded one RCT comparing demeclocycline to placebo in nine patients with schizophrenia who had intermittent hyponatremia and polydipsia. The effect of demeclocycline was nonsignificant in all analyses. There was also no significant difference in the number of episodes of serum sodium levels less than $125 \mathrm{mmol} / \mathrm{L}$ during the drug period (10 of 103 total sodium determinations) versus the placebo period. ${ }^{17}$ Given the small nature of this study and that the results showed no significant benefit over placebo, we do not recommend its use.
Our search did not yield any RCTs related to therapy with desmopressin acetate in the treatment of hyponatremia. Given its lack of use and the absence of published observational evidence, we cannot recommend that desmopressin acetate is safe for use in the treatment of hyponatremia.

\section{What are the accepted rules for sodium correction?}

Hyponatremia may develop acutely or on a chronic basis over weeks to months. Rapid correction of sodium concentration (especially in the chronic setting) may result in osmotic demyelination syndrome, brain injury and possibly death. Therefore, serum sodium correction with normal saline or through the introduction of a vasopressin receptor antagonist must not occur at more than $0.5 \mathrm{mmol} / \mathrm{h}$, or more than $12 \mathrm{mmol} / \mathrm{d}$. Patients should be in a setting where they can be easily monitored. The use of vasopressin receptor antagonists may be unfamiliar to many clinicians, and consideration should be given to defer their use to specialists who have more clinical experience. If the patient has serious symptoms of hyponatremia (e.g., seizures), then more rapid correction through the use of hypertonic saline may be necessary to prevent cerebral edema. ${ }^{38}$

\section{Gaps in knowledge}

Our systematic review highlighted a lack of evidence from RCTs for the management of hyponatremia. The emergence of RCTs to evaluate vasopressin receptor antagonists only served to emphasize the paucity of robust studies to evaluate all other types of management that are currently considered the standard of care for patients with hyponatremia. There is risk of considerable publication bias in evidence for the treatment of hyponatremia because of the large number of small industry-sponsored RCTs evaluating vasopressin receptor antagonists and a lack of other RCTs to evaluate other interventions.

Despite the risk of publication bias, there historically exists a "standard of care" or "consensus" about the management of hyponatremia despite the lack of a strong evidence base. It should also be emphasized that all RCTs comparing vasopressin receptor antagonists to placebo (or fluid restriction) all used the surrogate endpoint of changes in serum sodium concentration, and none had a primary outcome that was patient-focused. It is difficult for us to evaluate what the clinical significance is of changes in serum sodium of $2-5 \mathrm{mmol} / \mathrm{L}$ (as was reported in 
many of the vasopressin receptor antagonist studies). Currently, the evidence from clinical trials for vasopressin receptor antagonists suggests that they are safe with a low likelihood of adverse events; however, the lack of patient-focused outcomes suggests that they should not be widely used until more convincing evidence shows efficacy in improving outcomes. Furthermore, the cost associated with the use of vasopressin receptor antagonists (compared to current standard therapies) may also be seen as a potential barrier to the uptake of this intervention. It should also be emphasized that no study has shown that vasopressin receptor antagonists significantly reduce mortality.

\section{References}

1. Adrogué HJ, Madias NE. Hyponatremia. N Engl J Med 2000; 342:1581-6.

2. Flear CT, Gill GV, Burn J. Hyponatraemia: mechanisms and management. Lancet 1981;2:26-31.

3. Lauriat SM, Berl T. The hyponatremic patient: practical focus on therapy. J Am Soc Nephrol 1997;8:1599-607.

4. Miller M, Hecker MS, Friedlander DA, et al. Apparent idiopathic hyponatremia in an ambulatory geriatric population. J Am Geriatr Soc 1996; $44: 404-8$

5. Cárdenas A, Gines P, Marotta P, et al. Tolvaptan, an oral vasopressin antagonist, in the treatment of hyponatremia in cirrhosis. J Hepatol 2012;56:571-8.

6. Berl T, Quittnat-Pelletier F, Verbalis JG, et al. Oral tolvaptan is safe and effective in chronic hyponatremia. J Am Soc Nephrol 2010, 21:705-12.

7. Moher D, Liberati A, Tetzlaff J, et al.; The PRISMA Group. Preferred Reporting Items for Systematic Reviews and MetaAnalyses: The PRISMA Statement. Int J Surg 2010;8:336-41.

8. Chung HM, Kluge R, Shrier RW, et al. Clinical assessment of extracellular fluid volume in hyponatremia. Am J Med 1987;83: 905-8.

9. Yeates KE, Singer M, Morton AR. Salt and water: a simple approach to hyponatremia. CMAJ 2004;170:365-9.

10. Vaidya C, Ho W, Freda BJ. Management of hyponatremia: providing treatment and avoiding harm. Cleve Clin J Med 2010;77. 715-26.

11. Gross P. Treatment of hyponatremia. Intern Med 2008;47:885-91.

12. Goldman MB, Luchins DJ, Robertson GL. Mechanisms of altered water metabolism in psychotic patients with polydipsia and hyponatremia. N Engl J Med 1988;318:397-403.

13. Jose CJ, Perez-Cruet J. Incidence and morbidity of self-induced water intoxication in state mental hospital patients. Am J Psychiatry 1979;136:221-2

14. Verbalis JG, Goldsmith SR, Greenberg A, et al. Hyponatremia treatment guidelines 2007: expert panel recommendations. Am J Med 2007;120:S1-21.

15. Bartter FC, Schwartz WB. The syndrome of inappropriate secretion of antidiuretic hormone. Am J Med 1967;42:790-806.

16. Cherrill DA, Stote RM, Birge JR, et al. Demeclocycline treatment in the syndrome of inappropriate antidiuretic hormone secretion. Ann Intern Med 1975;83:654-6.

17. Alexander RC, Karp BL, Thompson S, et al. A double blind, placebo-controlled trial of demeclocycline treatment of polydipsiahyponatremia in chronically psychotic patients. Biol Psychiatry 1991;30:417-20.

18. Rose BD. New approach to disturbances in the plasma sodium concentration. Am J Med 1986;81:1033-40.

19. Soupart A, Gross P, Legros JJ, et al. Successful long term treatment of hyponatremia in syndrome of inappropriate antidiuretic hormone secretion with satavaptan (SR121463B), an orally active nonpeptide vasopressin V2-receptor antagonist. Clin J Am Soc Nephrol 2006;1:1154-60.

20. Gheorghiade M, Gottlieb SS, Udelson JE, et al. Vasopressin V2 receptor blockade with Tolvaptan versus fluid restriction in the treatment of hyponatremia. Am J Cardiol 2006;97:1064-7.

21. Rozen-Zvi B, Yahav D, Gheorghiade M, et al. Vasopressin receptor antagonists for the treatment of hyponatremia: systematic review and meta-analysis. Am J Kidney Dis 2010;56:325-37.

22. Verbalis JG, Zeltser D, Smith N, et al. Assessment of the efficacy and safety of intravenous conivaptan in patients with euvolaemic hyponatraemia: subgroup analysis of a randomized, controlled study. Clin Endocrinol (Oxf) 2008;69:159-68.

23. Annane D, Decaux G, Smith N. Efficacy and safety of oral conivaptan, a vasopressin-receptor antagonist, evaluated in a randomized, controlled trial in patients with euvolemic or hypervolemic hyponatremia. Am J Med Sci 2009;337:28-36.

24. Highlights of prescribing information: Vaprisol. Northbrook (IL): Astellas Pharma US. Available: www.astellas.us/docs /vaprisol.pdf (accessed 2012 Jan.)

25. Zeltser D, Rosansky S, van Rensburg H, et al.; Conivaptan Study Group. Assessment of the efficacy and safety of intravenous conivaptan in euvolemic and hypervolemic hyponatremia. Am J Nephrol 2007;27:447-57.

26. Kalra S, Efrati S, Arthur JM, et al. Effect of loading dose and formulation on safety and efficacy of conivaptan in treatment of euvolemic and hypervolemic hyponatremia. Am J Health Syst Pharm 2011:68:590-8.

27. Ghali JK, Koren MJ, Taylor JR, et al. Efficacy and safety of oral conivaptan: a VIA/V2 vasopressin receptor antagonist, assessed in a randomized, placebo-controlled trial in patients with euvolemic or hypervolemic hyponatremia. J Clin Endocrinol Metab 2006;91:2145-52.

28. Naidech AM, Paparello J, Leibling SM, et al. Use of conivaptan (Vaprisol) for hyponatremic neuro-ICU patients [published erratum in Neurocrit Care 2011;15:210]. Neurocrit Care 2010;13:57-61.

29. Josiassen RC, Goldman M, Jessani M, et al. Double-blind, placebo-controlled, multicenter trial of a vasopressin V2-receptor antagonist in patients with schizophrenia and hyponatremia. Biol Psychiatry 2008;64:1097-100.

30. Verbalis JG, Adler S, Schrier RW, et al.; SALT investigators. Efficacy and safety of oral tolvaptan therapy in patients with the syndrome of inappropriate antidiuretic hormone secretion. Eur J Endocrinol 2011;164:725-32.

31. Schrier RW, Gross P, Gheorghiade M, et al. Tolvaptan, a selective oral vasopressin V2-receptor antagonist, for hyponatremia. N Engl J Med 2006;355:2099-112.

32. Ginès $\mathrm{P}$, Wong $\mathrm{F}$, Watson $\mathrm{H}$, et al. Effects of satavaptan, a selective vasopressin V2 receptor antagonist, on ascites and serum sodium in cirrhosis with hyponatremia: a randomized trial. Hepatology 2008;48:204-13.

33. Aronson D, Verbalis JG, Mueller M, et al.; DILIPO investigators. Short- and long-term treatment of dilutional hyponatraemia with satavaptan, a selective arginine vasopressin V2-receptor antagonist: the DILIPO study. Eur J Heart Fail 2011;13:327-36.

34. Gerbes AL, Gulberg V, Gines P, et al. Therapy of hyponatremia in cirrhosis with a vasopressin receptor antagonist: A randomized double-blind multicenter trial. Gastroenterology 2003;124:933-9.

35. Abraham WT, Aranda JM, Boehmer JP, et al. Rationale and design of the treatment of hyponatremia based on lixivaptan in NYHA class III/IV cardiac patient evaluation (THE BALANCE) study. Clin Transl Sci 2010;3:249-53.

36. Rogers IR, Hook G, Stuempfle KJ, et al. An intervention study of oral versus intravenous hypertonic saline administration in ultramarathon runners with exercise-associated hyponatremia: a preliminary randomized trial. Clin J Sport Med 2011;21:200-3.

37. Katayama Y, Haraoka J, Hirabayashi H, et al. A randomized controlled trial of hydrocortisone against hyponatremia in patients with aneurysmal subarachnoid hemorrhage. Stroke 2007;38:2373-5.

38. Sterns RH, Riggs JE, Schochett SS. Osmotic demyelination syndrome following correction of hyponatremia. N Engl J Med 1986; 314:1535-42.

Affiliations: School of Medicine (Lee, Nistico, Yeates) Queen's University, Kingston, Ont.; Department of Medicine (Kilonzo), Kilimanjaro Christian Medical Centre, Moshi Tanzania.

Contributors: All of the authors contributed to the design and conception and to drafting and revising the article. All of the authors approved the final version submitted for publication. 